\title{
RF measurement technique as a tool to understanding the gas loading on automotive catalysts
}

\author{
David Kubinski ${ }^{1}$, Vladimir Malashchuk ${ }^{2}$ \\ ${ }^{1}$ Ford Research and Innovation Center, 2101 Village Rd., Dearborn, Michigan, 48121, USA, \\ ${ }^{2}$ Department of Functional Materials, University of Bayreuth, Bayreuth 95440, Germany \\ dkubinsk@ford.com
}

\begin{abstract}
:
The state of the oxygen storage material contained in automotive three-way catalysts (TWC's) influences their ability to oxidize hydrocarbons and $\mathrm{CO}$ while simultaneously reducing nitrogen oxides (NOx). It has been demonstrated that the RF-microwave resonant method offers the ability to directly measure the oxygen storage on the TWC's. Additionally, this technique has also been shown to be useful in other automotive applications such as measuring $\mathrm{NH}_{3}$ storage on diesel SCR catalysts, soot loading on particulate filters and NOx storage on traps. Here we give an overview of the various applications and present additional results for TWC's. We demonstrate how the frequency of the resonant mode and its corresponding quality factor vary both with the oxidation state of the TWC and its temperature. We compare the temperature dependencies of these parameters with the measured catalytic efficiency. We show that the imaginary component of the catalyst's dielectric permittivity, determined from the quality factor of the $\mathrm{TE}_{111}$ resonant mode, exhibits a large increase near the temperature at which the catalyst begins change its oxidation state with gas composition.
\end{abstract}

Key words: Microwave, resonant cavity, automotive three-way catalyst, gas storage.

\section{Introduction}

Optimization of the oxidation state of the oxygen storage material contained in an automotive three-way catalyst (TWC), typically a ceria oxide, is essential for ideal emission control. A fully oxidized TWC can result in NOx emissions, while one depleted can produce hydrocarbon and $\mathrm{CO}$ emissions. Knowledge of the state of oxygen storage of the TWC is typically inferred through use of upstream and downstream oxygen sensors. It has been demonstrated that the RF-microwave resonant technique offers an alternative approach, enabling a direct measure of the storage state via measurement of the TWC's dielectric properties which change with gas loading [1]. However, knowledge of this technique's temperature dependency is required to correctly determine the oxidation state of the TWC.

\section{Experimental}

Figure 1 shows the configuration of the cylindrical microwave resonant cavity used to measure the oxygen storage on the TWC. The TWC, cored from a larger piece used in serial application, was mounted near the center of the cavity. Two antennae, each positioned between the TWC and the stainless screens at each end of the cavity, enabled measurement of the complex S12 transmission parameter. From this the real and imaginary components of the dielectric permittivity were calculated following the procedure described in [2]. The microwave signals were generated and detected by an Agilent E5071C network analyzer. A 60 liter/min heated gas flow was used with temperature probes placed at the ends of the cavity. A FTIR gas analyzer and a $\mathrm{H}_{2}$ mass spectrometer monitored the composition of the outlet gas.

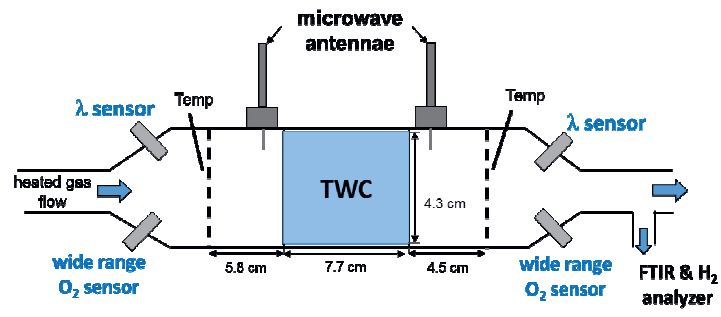

Fig. 1. Schematic of the cylindrical resonant cavity and the TWC placed within. Screens, shown by the dashes lines, are placed both up and downstream as are thermocouples and oxygen sensors. 
The gas was cycled between two compositions: One an oxidizing composition ("lean", $\lambda \approx 1.01$ ) and the other a reducing gas mixture ("rich", $\lambda \approx 0.99$ ). Each constant concentration gas step lasted 3 minutes, sufficiently long to fully oxidize (when "lean") or reduce (when "rich") the TWC's oxygen storage material. Cycling of the gases continued as the TWC temperature was ramped to $600^{\circ} \mathrm{C}$ in 180 minutes. The change in oxidation state of the TWC after each "lean" to "rich" gas step was calculated from analysis of the downstream gas concentrations.

\section{Results}

Figure 2 shows temperature dependencies of both the resonant frequency, $f_{o}$, and the reciprocal quality factor, $Q^{-1}$, for the $T E_{111}$ resonant mode. These parameters were chosen since the frequency is related to the real component of the dielectric permittivity, whereas the reciprocal quality factor relates to the imaginary [2]. Plotted are the average values obtained near the end of each "lean" and "rich" gas step. The behavior below $\approx 150^{\circ} \mathrm{C}$ has been shown to be due to the desorption of water from the TWC.
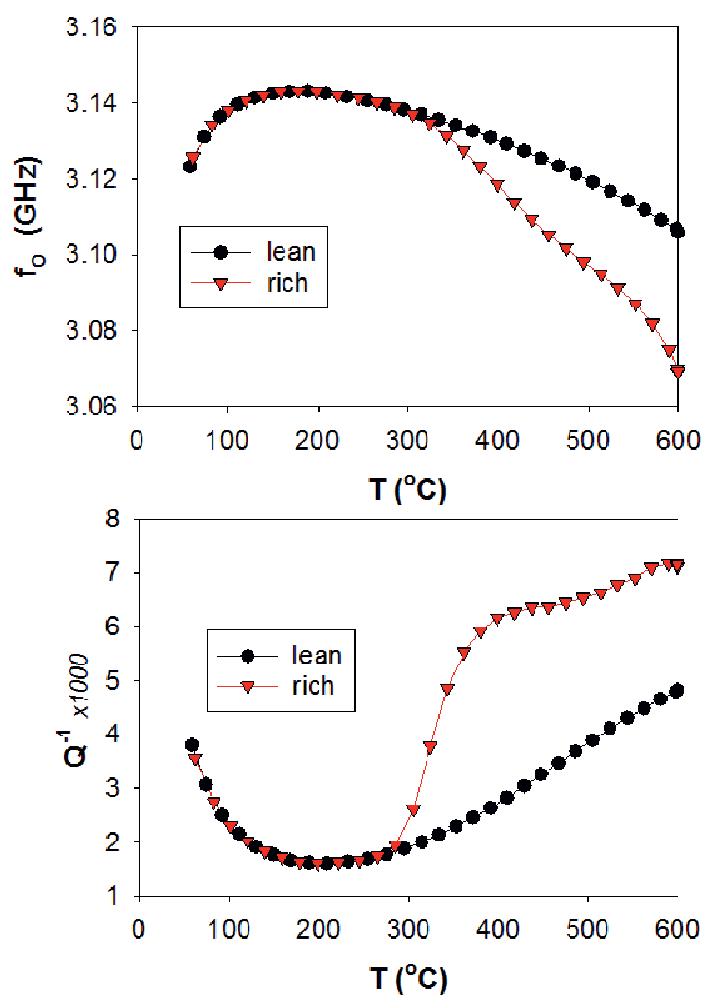

Fig. 2. Temperature dependency of both the resonant frequency and the corresponding inverse quality factor for the $T E_{111}$ resonant mode. Data in black were taken when the TWC was fully oxidized. Data in red were taken after the oxygen storage was depleted by a reducing gas stream.
Figure 3 plots the corresponding temperature dependency of the total change in oxygen storage of the TWC, $\Delta$ (oxygen storage), determined for each "lean" to "rich" gas step. These values were calculated from the measured variation in gas concentrations downstream of the TWC during the "rich" gas steps as the oxygen storage was depleted. We note the similarities of the curve in Fig. 3 with the difference between the $Q^{-1}$ curves in Fig. 2 . Near the temperature at which the TWC begins to change its oxidation state (after from a "lean" to a "rich" gas switch), $\approx 290^{\circ} \mathrm{C}, \Delta \mathrm{Q}^{-1}$ (the difference between "rich" and "lean" $Q^{-1}$ values) also exhibits a similar sharp rise with increasing temperature.

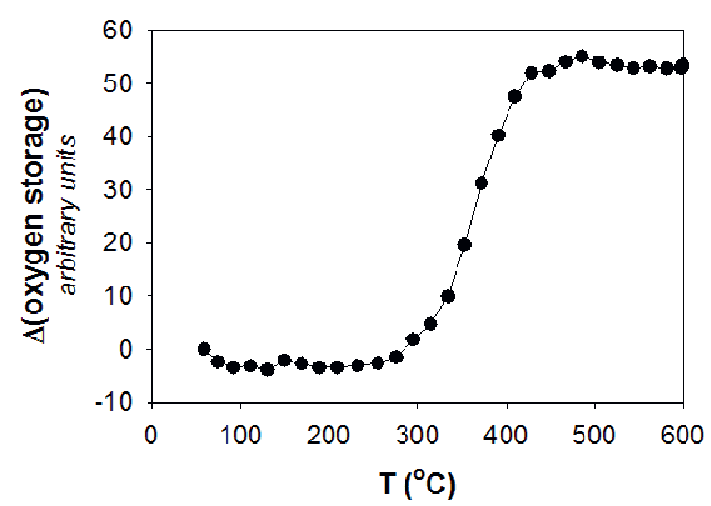

Fig. 3. Total change in oxygen storage of the TWC calculated for the "lean" to "rich" gas steps. Data are plotted as a function of temperature. The $\Delta$ (oxygen storage) units are arbitrary and are relative to the value at $\approx 50^{\circ} \mathrm{C}$.

These findings demonstrate the temperature dependency of the dielectric losses for a "lean" to "rich" gas step, determined from $\Delta Q^{-1}$, correlates well with the onset of the change in oxygen storage on the TWC. Additionally, the curves shown in Fig. 2 can be used compensate for the influence of temperature, and thus properly determine the state of the oxygen storage material on the TWC.

\section{References}

[1] R. Moos, M, Wedemann, M. Sporl, S. Reiß, G. Fischerauer, Direct Catalyst Monitoring by Electrical Means: An Overview on Promising Novel Principles, Top Catal 52:2035-2040 (2009); DOI 10.1007/s11244-009-9399-6

[2] M. Dietrich, C. Jahn, P. Lanzerath, R. Moos, Microwave-Based Oxidation State and Soot Loading Determination on Gasoline Particulate Filters with Three-Way Catalyst Coating for Homogenously Operated Gasoline Engines, Sensors 15, 21971-21988 (2015); doi:10.3390/s1509 